\title{
Microscopy: The Gateway to Bio-Inspired Design Innovation
}

\author{
Sue Okerstrom ${ }^{1}$
}

1. Lichen Labs LLC, Minneapolis, MN, USA

New challenges and opportunities for microscopists lie in the emerging fields of biomimicry and bioinspired design. Organisms in nature have developed structures, processes, and materials to adapt to the context of their environment over the 3.8 billion years of life on earth. Biomimetic designs, including materials and surfaces, are created by studying and emulating the processes and structures found at the micro and nanoscale in nature that play a vital role in the performance of the organism. Strategies and principles of efficiency and effectiveness used by successful species in nature could be followed in creating innovative sustainable materials. An example is the self-cleaning properties of the leaves of the lotus flower in muddy water, dubbed the "lotus effect" [1]. Dirt particles are picked up by water droplets which roll off the ultra-hydrophobic micro and nano-textured surface of the leaf.

Surprisingly, the vast majority of studies in biomimetics have been in fields of chemistry, materials, and engineering. This interdisciplinary pursuit would benefit significantly by teaming up with biologists [2], and microscopists.

The physical sciences could benefit from functional biological research on adaptations. An organism may have unique adaptations such as stripes on the zebra, whose primary function is to deter biting flies. The stripes interfere with landings of the flies at close range; however, the mechanism is still not understood [3].

The biologist would be aided by the research approach of the materials scientist microscopist. The study of nature is approached as one would examine a competitive device or perform failure analysis. The process starts with taking photos of the organism on the macro scale, if possible, in its natural environment. Drawings and observations are noted as to the conditions, structure, behavior, and interactions. Next, the whole organism or portions of interest, are examined in a stereo (dissecting) microscope. For analysis in a variable pressure scanning electron microscope (SEM), Insects, feathers, plant parts can be mounted on a carbon adhesive pad, or as this orb-weaving spider, with its web and egg in a plastic jar. Large specimens can be examined at a long working distance to visualize the macro view and possibly systems interactions, as the spider with its foot attached by $\sim 1 \mathrm{~nm}$ threads to the dragline of the web presumably to improve sensing and response time to catch unsuspecting prey (Figure 1).

The ability to zoom in and zoom out is critical to elucidate the functions of structures observed. Determining the strategies and mechanisms of how the adaptation works are key steps in the emulation process. Structural components can be measured and drawn - developing a "blueprint" of how that adaptation works that can be utilized to build a design that meets similar human challenges.

Examples will be presented utilizing this "biology to design" discovery process. Currently, the physical sciences are dominating the biomimetic field. Generally, they are addressing a specific challenge inspired by just the feature or process of interest. A better understanding of the complex biology would move down the path from inspiration toward more fully emulating nature's properties. These properties include materials and energy-saving strategies, non-toxic chemistries, self-healing abilities, adaptive designs, and no waste recycling systems. 
Multi-functional teams of engineers, chemists, physicists, biologists, and designers are needed to analyze living devices and systems and learn their innovative sustainable strategies. Emulating nature's materials, structures, systems, and processes are the future if we are to have one. Microscopy is the gateway to this process.

\section{References:}

[1] W Barthlott and C. Neinhuis, Planta 202, (1997) p.1-8.

[2] E Snell-Rood, Nature 529 (2016), p.277-278.

[3] T Caro et al, PLOS ONE, [Online] 14, 2 (2019),https://doi.org/10.1371/journal.pone.0210831
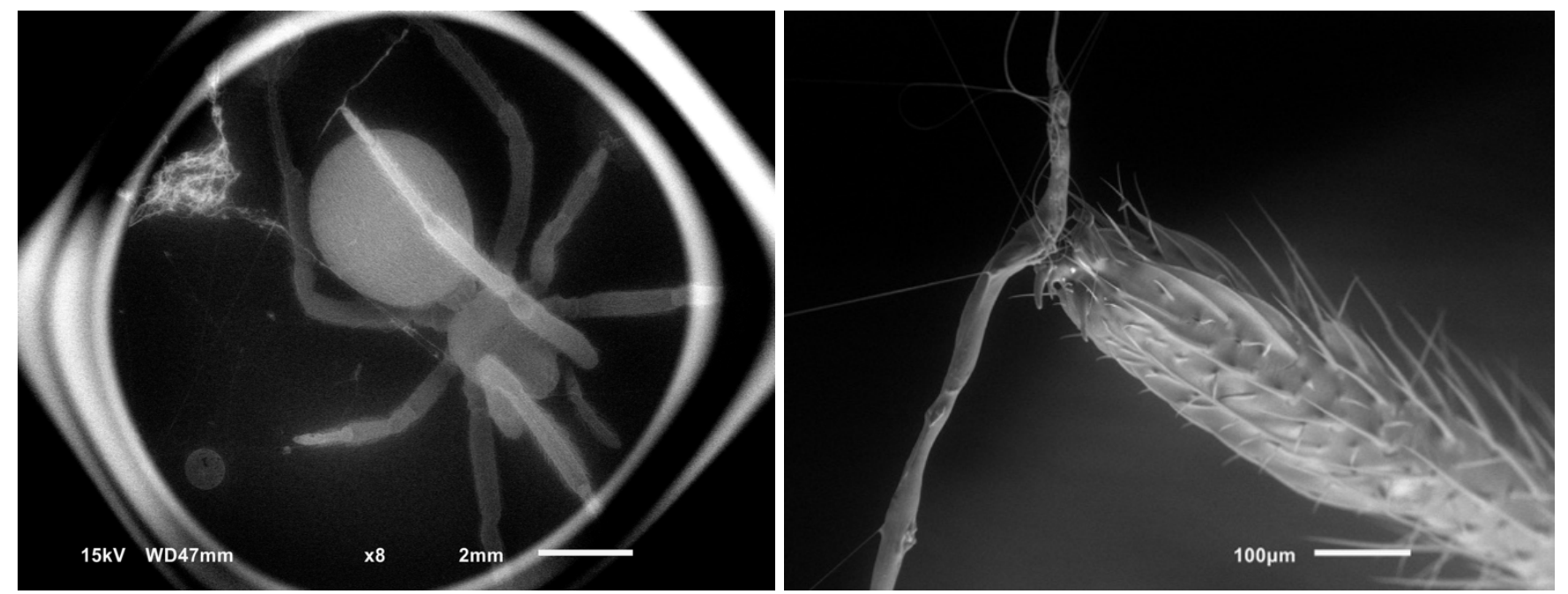

Figure 1. An orb weaver spider in a plastic jar with web and egg at left with the magnified foot of the spider attached via $1 \mathrm{~nm}$ threads to the dragline of its web at right as viewed in a JEOL JSM-6010 plus L/A variable pressure SEM. 